\title{
An unusual anatomical variation of the inferior alveolar nerve
}

\author{
Shogo Maekawa ${ }^{1,2,3}$, Mizuki Nagata ${ }^{4}$, Yuki Matsushita ${ }^{4,5}$, R. Shane Tubbs ${ }^{6,9,10,11}$, Joe Iwanaga ${ }^{6,7,8}$ \\ ${ }^{1}$ Department of Periodontics and Oral Medicine, University of Michigan School of Dentistry, Ann Arbor, MI, ${ }^{2}$ Biointerfaces Institute, North Campus \\ Research Complex, University of Michigan, Ann Arbor, MI, USA, ${ }^{3}$ Department of Periodontology, Graduate School of Medical and Dental Sciences, \\ Tokyo Medical and Dental University, Tokyo, Japan, ${ }^{4}$ Department of Orthodontics and Pediatric Dentistry, University of Michigan School of Dentistry, \\ Ann Arbor, MI, USA, ${ }^{5}$ Department of Clinical Oral Oncology, Nagasaki University Graduate School of Biomedical Sciences, Nagasaki, Japan, \\ ${ }^{6}$ Department of Neurosurgery, Tulane Center for Clinical Neurosciences, Tulane University School of Medicine, New Orleans, LA, USA, ${ }^{7}$ Dental and \\ Oral Medical Center, Kurume University School of Medicine, Fukuoka, ${ }^{8}$ Division of Gross and Clinical Anatomy, Department of Anatomy, Kurume \\ University School of Medicine, Fukuoka, Japan, ${ }^{9}$ Department of Neurosurgery and Ochsner Neuroscience Institute, Ochsner Health System, New \\ Orleans, LA, USA, ${ }^{10}$ Department of Anatomical Sciences, St. George's University, St. George's, Grenada, ${ }^{11}$ Department of Structural \& Cellular Biology, \\ Tulane University School of Medicine, New Orleans, LA, USA
}

Abstract: A number of studies have previously shown variations of inferior alveolar, however, only a few reports focused on nearby the foramen ovale. In a formalin fixed cadaver, we identified three minor branches (anterior, middle, and posterior branches) arising from the main trunk of the mandibular nerve adjacent to the foramen ovale, passing lateral to the maxillary artery (MA), and joining the inferior alveolar nerve. The diameter of the branches was $0.68 \mathrm{~mm}, 1.43 \mathrm{~mm}$, and $0.40 \mathrm{~mm}$, respectively. The branches traveled inside the lateral pterygoid muscle (LPM) or between the LPM and tensor/levator veli palatini. Moreover, all of the branches were superficial to MA. Knowledge of such a variation might be helpful to dentists during, for example, anesthetic blockade and various oral surgeries.

Key words: Cadaver, Clinical anatomy, Inferior alveolar nerve, Lingual nerve, Mandibular nerve

Received June 9, 2020; Revised July 14, 2020; Accepted July 30, 2020

\section{Introduction}

The inferior alveolar nerve (IAN) is a large branch of the mandibular nerve and arises from the nerve below the foramen ovale to descend through the infratemporal fossa (ITF) to reach the pterygomandibular space. Before entering the mandibular foramen, the IAN gives rise to the nerve to mylohyoid. Communications between the IAN, lingual nerve (LN), hypoglossal nerve or the auriculotemporal nerve (ATN) have been reported [1-5]. The IAN is clinically important nerve, especially in dentistry so that inferior alveolar nerve

\section{Corresponding author:}

Joe Iwanaga (10)

Department of Neurosurgery, Tulane Center for Clinical Neurosciences, Tulane University School of Medicine, New Orleans, LA 70112, USA E-mail: iwanagajoeca@gmail.com blockade (IANB) is better understood [6]. We report a presumably uncommon anatomical variation of the IAN adjacent to the foramen ovale.

\section{Case Report}

During routine dissection, a variant IAN was found in a formalin-fixed Caucasian male cadaver who was 74-yearsold at death. In the left infratemporal fossa, three minor branches (anterior, middle, and posterior branches) arose from the main trunk of the mandibular nerve, passed lateral to the maxillary artery (MA), and joining the IAN (Fig. 1). The anterior and middle branches traveled through the lateral pterygoid muscle (LPM) and the posterior branches traveled between the LPM and tensor/levator veli palatini. The middle and posterior branches arose from the mandibular nerve as a common trunk and then soon bifurcated into two

\section{Copyright ( $\odot 2020$. Anatomy \& Cell Biology}

This is an Open Access article distributed under the terms of the Creative Commons Attribution Non-Commercial License (http://creativecommons.org/licenses/by-nc/4.0/) which permits unrestricted non-commercial use, distribution, and reproduction in any medium, provided the original work is properly cited. 

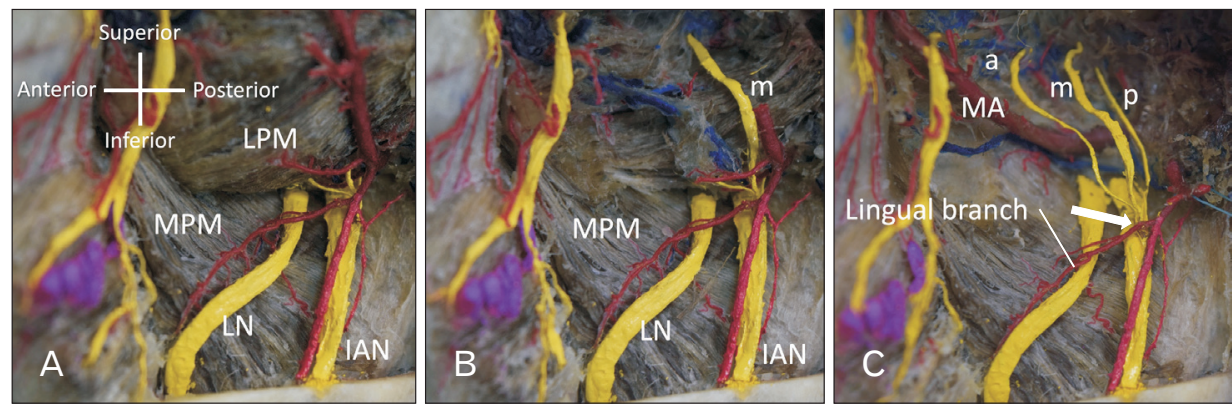

Fig. 1. Lateral view of the three variant branches arising from mandibular nerve in the left infratemporal fossa. The upper half of the mandibular ramus has been removed. (A) The three minor branches are not shown. (B) The middle branch (m) running within the lateral pterygoid muscle is shown. (C) The a, $\mathrm{m}$, and $\mathrm{p}$ branches are shown after removal of the lateral pterygoid muscle. Note the three branches forming a common trunk to join the IAN at the root of the lingual branch of the inferior alveolar artery (arrow). a, anterior; IAN, inferior alveolar nerve; LPM, lateral pterygoid muscle; m, middle; MA, maxillary artery; MPM medial pterygoid muscle; p, posterior.

branches. Interestingly, the three branches united and joined the IAN at the root of the lingual branch of the inferior alveolar artery (IAA) and slightly touched the lingual branch. In addition, two, small contributions that originated from a ganglion-like structure, joined the common trunk of the middle and posterior branches, and the posterior branch (Fig. 2). The diameter of the anterior, middle, and posterior branches was $0.68 \mathrm{~mm}, 1.43 \mathrm{~mm}$, and $0.40 \mathrm{~mm}$, respectively. No other variations were observed in the maxillofacial and neck regions of this cadaver. The IAN on the contralateral side was normal. No scar was found in the head and neck regions. Histological observations were not conducted in this study.

\section{Discussion}

The IAN is one of main trunks of mandibular nerve. The minor three branches we found were oriented from mandibular nerve and connected to IAN. In 2004, Kim et al. [1] classified the branching of the IAN and LN into four types. Type IV was a complex form of branches of the mandibular nerve and its frequency was $6.3 \%$. Iamsaard et al. [2] reported a unique connection between the trunk of the mandibular nerve and the LN communication. Loughner et al. [7] reported three of 52 specimens with three branches of the mandibular nerve that were apparently entrapped in the lateral pterygoid muscle, i.e., anterior and posterior deep temporal nerves and masseteric nerve. These three nerves, in the present case, were resected before dissecting the lateral pterygoid muscle.

Embryologically, the first pharyngeal arch is developed at the 6 th week of gestation. Thereafter, branches of the

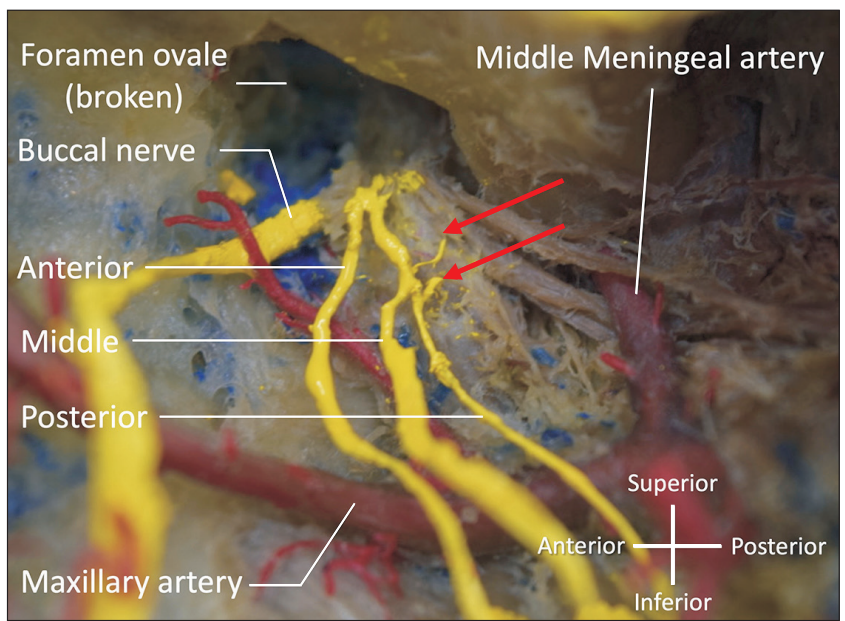

Fig. 2. A magnified image adjacent to the foramen ovale. Note the two small branches from the ganglion-like structure (arrows).

mandibular nerve including the IAN, LN and ATN develop. Anil et al. [8] reported a communicating branch between the ATN and IAN and the MA coursed between the IAN and communicating branch, which appeared to be entrapped. Wolf et al. [9] reported a similar case and described an IAN split by the MA. Moreover, Khan et al. [10] reported three similar cases (out of 50) of a MA splitting the IAN These reported cases have some similar features to the present case. Although three branches found in the present study were very small in diameter, as some of these could distribute mental region via the mental nerve, compression of the nerves by LPM contraction might result in dysfunction of the mental nerve. In addition, the lingual branch of the IAA slightly contacted three branches at its root. This might result in ischemia of the LN as the lingual branch of the IAA supply the LN [11]. 
In dentistry, IANB is most commonly performed when treating the mandibular molar teeth. In 1973, the GowGates [12] technique, a modified technique of the IANB, was published to target the main trunk of the mandibular nerve. The Vazirani-Akinosi technique is also an alternative option of a modified IANB [13]. Direct injury of variant branches such as the ones found in the present case might occur with either the Gow-Gates or Vazirani-Akinosi techniques due to the higher position of the needle insertion than the regular IANB technique.

A tiny branch between the otic ganglion and common trunk of the middle and posterior branches was also observed. Although postganglionic parasympathetic fibers are not mentioned for the IAN, it might be possible that postganglionic parasympathetic fibers travel with the IAN to be mental nerve and innervate the lower labial glands [14]. Another potential purpose of this fiber might be to join the nerve to mylohyoid to innervate the submandibular gland as the nerve to the mylohyoid runs near the submandibular gland.

In conclusion, we found three contributions from the mandibular nerve connecting to the IAN. These all traveled inside the LPM or between the LPM and tensor/levator veli palatini and all of them were superficial to the MA. Knowledge of such a variation might be helpful to dentists during, for example, anesthetic blockade and various oral surgeries.

\section{ORCID}

Shogo Maekawa: https://orcid.org/0000-0002-6207-7826

Mizuki Nagata: https://orcid.org/0000-0003-3874-2846

Yuki Matsushita: https://orcid.org/0000-0001-9577-3249

R. Shane Tubbs: https://orcid.org/0000-0003-1317-1047

Joe Iwanaga: https://orcid.org/0000-0002-8502-7952

\section{Author Contributions}

Conceptualization: RST, JI. Data acquisition: YM, RST, JI. Data analysis or interpretation: SM, MN, JI. Drafting of the manuscript: SM, MN, YM. Critical revision of the manuscript: RST, JI. Approval of the final version of the manuscript: all authors.

\section{Conflicts of Interest}

No potential conflict of interest relevant to this article was reported.

\section{Acknowledgements}

The authors appreciate those who donated their body for anatomical study and research.

\section{References}

1. Kim SY, Hu KS, Chung IH, Lee EW, Kim HJ. Topographic anatomy of the lingual nerve and variations in communication pattern of the mandibular nerve branches. Surg Radiol Anat 2004;26:128-35.

2. Iamsaard S, Singsorn J, Boonruangsri P. An unusual communication between the trunk of the mandibular nerve and the lingual nerve in a female cadaver. Acta Med Acad 2015;44:2012.

3. Muraleedharan A, Veeramani R, Chand P. Variations in the branching pattern of posterior division of mandibular nerve: a case report. Surg Radiol Anat 2014;36:947-50.

4. Carter RB, Keen EN. The intramandibular course of the inferior alveolar nerve. J Anat 1971;108(Pt 3):433-40.

5. Shimotakahara R, Lee H, Mine K, Ogata S, Tamatsu Y. Anatomy of the lingual nerve: application to oral surgery. Clin Anat 2019;32:635-41.

6. Khoury J, Mihailidis S, Ghabriel M, Townsend G. Anatomical relationships within the human pterygomandibular space: relevance to local anesthesia. Clin Anat 2010;23:936-44.

7. Loughner BA, Larkin LH, Mahan PE. Nerve entrapment in the lateral pterygoid muscle. Oral Surg Oral Med Oral Pathol 1990;69:299-306.

8. Anil A, Peker T, Turgut HB, Gülekon IN, Liman F. Variations in the anatomy of the inferior alveolar nerve. Br J Oral Maxillofac Surg 2003;41:236-9.

9. Wolf KT, Brokaw EJ, Bell A, Joy A. Variant inferior alveolar nerves and implications for local anesthesia. Anesth Prog 2016;63:84-90.

10. Khan MM, Darwish HH, Zaher WA. Perforation of the inferior alveolar nerve by the maxillary artery: an anatomical study. $\mathrm{Br}$ J Oral Maxillofac Surg 2010;48:645-7.

11. Harn SD, Durham TM. Anatomical variations and clinical implications of the artery to the lingual nerve. Clin Anat 2003;16:294-9.

12. Gow-Gates GA. Mandibular conduction anesthesia: a new technique using extraoral landmarks. Oral Surg Oral Med Oral Pathol 1973;36:321-8.

13. Haas DA. Alternative mandibular nerve block techniques: a review of the Gow-Gates and Akinosi-Vazirani closed-mouth mandibular nerve block techniques. J Am Dent Assoc 2011;142 Suppl 3:8S-12S.

14. Iwanaga J, Saga T, Tabira Y, Nakamura M, Kitashima S, Watanabe K, Kusukawa J, Yamaki K. The clinical anatomy of accessory mental nerves and foramina. Clin Anat 2015;28:848-56. 